\title{
Oridonin induces NPM mutant protein translocation and apoptosis in NPM1c+ acute myeloid leukemia cells in vitro
}

\author{
Fei-fei $\mathrm{LI}^{2, \#}$, Sha $\mathrm{YI}^{1, \#}$, Lu WEN ${ }^{1}$, Jing HE${ }^{1}$, Li-jing YANG ${ }^{1}$, Jie ZHAO ${ }^{1}$, Ben-ping ZHANG ${ }^{1}$, Guo-hui CUI ${ }^{1, *}$, Yan CHEN ${ }^{1, *}$ \\ ${ }^{1}$ Department of Hematology, Union Hospital, Tongji Medical College, Huazhong University of Science and Technology, Wuhan \\ 430022, China; ${ }^{2}$ Department of Cardiovascular Surgery, Union Hospital, Tongji Medical College, Huazhong University of Science and \\ Technology, Wuhan 430022, China
}

Aim: Skewed cytoplasmic accumulation of NPM mutant protein (NPM1c+) is close related to leukemia pathogenesis. The aim of this study was to investigate whether oridonin, a diterpenoid isolated from the Chinese traditional medicine Rabdosia rubescens, was able to interfere with NPM1c+ protein trafficking and induce apoptosis in NPM1c+ acute myeloid leukemia cells in vitro.

Methods: OCI-AML3 cell line harboring a NPM1 gene mutation was examined. Cell growth was detected by MTT assay. Cell apoptosis was evaluated using flow cytometry and Hoechst 33258 staining. The expression and subcellular localization of relevant proteins were detected by Western blot and immunofluorescent staining. The mRNA expression was detected by RT-PCR.

Results: Oridonin $(2-12 \mu \mathrm{mol} / \mathrm{L}$ ) dose-dependently inhibited the viability of OCl-AML3 cells (the IC 50 value was $3.27 \pm 0.23 \mu \mathrm{mol} / \mathrm{L}$ at 24 h). Moreover, oridonin induced OCl-AML3 cell apoptosis accompanied by activation of caspase-3 and nuclear translocation of NPM1c+ protein. Oridonin did not change the expression of Crm1 (the export receptor for nuclear export signal-containing proteins), but induced nuclear translocation of Crm1. Oridonin markedly increased the expression of nucleoporin98 (Nup98), which had an important role in Crm1-mediated nuclear protein export, and induced nuclear accumulation of Nup98. Furthermore, oridonin markedly increased the expression of p14arf and p53.

Conclusion: In NPM1c+ leukemia cells, oridonin induces NPM1c+ protein translocation into the nucleus possibly via nuclear accumulation of Crm1; the compound markedly increases p53 and p14arf expression, which may contribute to cell apoptosis.

Keywords: oridonin; acute myeloid leukemia; NPM mutation; protein trafficking; Crm1; nucleoporin98; apoptosis; p53; p14arf

Acta Pharmacologica Sinica (2014) 35: 806-813; doi: 10.1038/aps.2014.25

\section{Introduction}

Acute myeloid leukemia (AML) is an aggressive malignancy characterized by significant clinical and molecular heterogeneity. Over the past two decades, genetic analysis and molecular biology have revealed the mechanisms of leukemogenesis and have markedly improved prognostic stratification of AML patients. In 2005, Falini et al identified heterozygous nucleophosmin (NPM)1 mutations as the most common genetic lesion in adult AML (with $60 \%$ of cases having a normal karyotype $)^{[1]}$, which was subsequently recognized as a provisional entity in the new 2008 WHO classification ${ }^{[2]}$.

\footnotetext{
\#These two authors contributed equally to this work.

* To whom correspondence should be addressed.

E-mail yanchen981@aliyun.com (Yan CHEN); ghcui@medmail.com.cn (Guo-hui CUI)

Received 2013-12-14 Accepted 2014-03-24
}

Approximately 50 NPM1 mutations have been identified to date, which all cause the skewed cytoplasmic accumulation of the NPM mutant protein (NPM1c+) and were thought to be responsible for leukemia pathogenesis ${ }^{[3]}$.

In a mouse model, the overexpression of cytoplasmic NPMmut created a proliferative advantage in the myeloid lineage ${ }^{[4]}$, while in zebrafish, the expression of the cytoplasmic NPMmut resulted in the expansion of hematopoietic cells ${ }^{[5]}$. All mutations lead to two critical changes at the NPM1 protein C-terminus, including the loss of tryptophan residues 288 and 290 (which prevent NPM1 binding in the nucleolus) ${ }^{[6]}$ and the generation of a new NES (nuclear export signal) motif (which reinforces the Crm1-dependent nuclear export of the NPM1 protein ${ }^{[7]}$. Several lines of evidence point to altered Crm1dependent nucleophosmin trafficking as the critical event in leukemogenesis ${ }^{[3]}$. Therefore, altered nucleophosmin trafficking in AML is an attractive therapeutic target. 
Herbal medicines and medicines derived from herbs have been used as alternative treatments worldwide for thousands of years ${ }^{[8]}$. There is growing interest in the possible use of natural products for the treatment of malignant disease and their potential for combination with current effective drugs ${ }^{[9]}$.

Oridonin is an example of a natural product recently described to have potential as an inhibitor of carcinogenesis and inflammation ${ }^{[10]}$. Oridonin is a plant-derived diterpenoid isolated from Rabdosia rubescens that is most commonly used as a Chinese traditional medicine. Until now, the potential use of oridonin as a cancer chemotherapeutic agent has been suggested in several cancers, including colon cancer ${ }^{[11,12]}$, pancreatic cancer ${ }^{[13]}$, fibrosarcoma ${ }^{[14]}$ and hepatocellular carcinoma $^{[15]}$. In addition, other reports have demonstrated that oridonin exhibits significant inhibitory effects on hematological malignancies, including NB4 ${ }^{[16]}$, HL-60 ${ }^{[17]}$, U937, kausima-1, K562 and OCIM2 ${ }^{[18,19]}$ leukemia cells, primary AML cells ${ }^{[19]}$ and multiple myeloma RPMI8266 cells ${ }^{[20]}$. Although Zhou et al found that oridonin induced apoptosis in $\mathrm{t}(8 ; 21)$ AML cells through the targeting of the AML1-ETO (AE) fusion protein, which plays a critical role in leukemogenesis ${ }^{[19,21]}$, the effect of oridonin on NPM1c+ leukemia cells and the precise molecular mechanisms are still unknown.

We investigated if oridonin was able to influence the expression and subcellular localization of NPM1 and to induce apoptosis and the possible underlying mechanism in OCIAML3 cells (known to harbor a NPM1 gene mutation), which are a promising preclinical model for screening compounds designed to interfere with the NPM-altered nucleo-cytoplasmic trafficking. Therefore, we evaluated the growth inhibitory effect, processing of caspases, cleavage of PARP, and expression or subcellular localization of NPM-mut and NPMwt. Furthermore, we examined the potential of oridonin to interfere with the expression and subcellular localization of Crm1, which are considered to be the critical events in altered nucleo-cytoplasmic trafficking.

\section{Materials and methods Materials}

Oridonin was purchased from Sigma-Aldrich (St Louis, MO, USA) and initially dissolved in dimethyl sulfoxide (DMSO) to make a stock solution. The DMSO concentration was maintained below $0.1 \%$ in cell culture and did not exert any detectable effect on cell growth or cell death. DMSO, trypan blue, Hoechst 33258 dye, 3-(4,5-dimethylthiazol-2-yl)-2,5-diphenyltetrazoliumbromide (MTT) and propidium iodide (PI) were purchased from Sigma-Aldrich (St Louis, MO, USA). The NPM-mut specific antibody was kindly provided by Brunangelo Falini (Perugia, Italy). The NPM-wt specific antibody was obtained from Invitrogen (Carlsbad, CA, USA). Primary antibodies for caspase-3, PARP, nucleoporin98 (Nup98) and p14arf were purchased from Cell Signaling Technology (Danvers, MA, USA). Crm1 and p53 antibodies were purchased from Santa Cruz Biotechnology (Santa Cruz, CA, USA). LaminB1 was purchased from the Proteintech Group (Chicago, IL, USA). The $\gamma$-tubulin antibody was purchased from Jackson
ImmunoResearch (West Grove, PA, USA).

\section{Cell culture}

The AML $\left(\mathrm{FAB} \mathrm{M}_{4}\right)$ cell line OCI-AML3 ${ }^{[22]}$ with a NPM mutation was kindly provided by MD MINDEN (Ontario Cancer Institute, Toronto, ON, Canada). Cells were grown in RPMI1640 medium (GIBCO, Gaithersburg, MD, USA) supplemented with 10\% fetal bovine serum (FBS) (Shijiqing, HangZhou, China). Cultures were maintained in a humidified atmosphere with $5 \% \mathrm{CO}_{2}$ at $37^{\circ} \mathrm{C}$.

\section{Cell growth inhibition assay}

The inhibition of cell growth was determined by MTT assay. Cells $\left(1 \times 10^{4}\right.$ per well) were exposed to various concentrations of oridonin (0-12 $\mu \mathrm{mol} / \mathrm{L})$ for 24,48 , and $72 \mathrm{~h}$ in 96-well plates. Thereafter, MTT (5 mg/mL in PBS) was added to each well for $4 \mathrm{~h}$, the supernatant was discarded and DMSO was added. Once the blue crystals were dissolved, the optical density $(O D)$ was measured at $490 \mathrm{~nm}$ using a plate microreader (Biotech Instruments, NY, USA). The percentage of cell growth inhibition was calculated as follows: cell inhibitory ratio $(\%)=[1-(O D$ of the experimental samples $/ O D$ of the control $)] \times 100 \%(n=3$, mean $\pm S D)$. The half maximal inhibitory concentration $\left(\mathrm{IC}_{50}\right)$ was defined as the oridonin concentration that caused a $50 \%$ inhibition of cell proliferation.

\section{Flow cytometry analysis for apoptosis}

The cells were seeded in 6 -well plates $\left(5 \times 10^{5} / \mathrm{mL}\right)$ and were treated with oridonin for $24 \mathrm{~h}$. The cells were harvested and washed with cold PBS, and then resuspended in $100 \mu \mathrm{L}$ of binding buffer. Subsequently, phosphatidyl serine on the surface of apoptotic cells was detected using Annexin V/FITC and a PI apoptosis detection kit following the manufacturer's instructions (Bender MedSystems Inc, Burlingame, CA, USA). The number of cells undergoing apoptosis was evaluated by FACS flow cytometry (BD, San Diego, CA, USA). A population of at least 10000 cells was analyzed.

\section{Hoechst 33258 staining assay}

Cells $\left(5 \times 10^{5} / \mathrm{mL}\right)$ were treated with oridonin for $24 \mathrm{~h}$. Cells were collected, washed and then fixed for $10 \mathrm{~min}$ before deposition onto polylysine-coated coverslips. Subsequently, the samples were permeabilized with $0.25 \%$ Triton X-100 for 5 min and stained with $1 \mu \mathrm{g} / \mathrm{mL}$ Hoechst 33258 for $30 \mathrm{~min}$ at $37^{\circ} \mathrm{C}$. The slides were mounted with glycerol-PBS, and the images were captured and visualized using a FV500 confocal microscope (Olympus, Tokyo, Japan).

\section{Western blot analysis}

The OCI-AML3 cells $\left(2 \times 10^{6}\right)$ were seeded and treated with different concentrations of oridonin for $24 \mathrm{~h}$. Cells were lysed in RIPA buffer $(50 \mathrm{mmol} / \mathrm{L}$ Tris- $\mathrm{HCl}, \mathrm{pH} 7.4 ; 150 \mathrm{mmol} / \mathrm{L}$ $\mathrm{NaCl} ; 1 \% \mathrm{NP}-40 ; 0.5 \%$ sodium deoxycholate; $0.1 \%$ SDS) containing a complete protease inhibitor cocktail and PMSF (Calbiochem, San Diego, USA). Nuclear and cytoplasmic protein was extracted according to the manufacturer's instructions for 
the nuclear and cytoplasmic extraction reagents (Beyotime, Haimen, China). Homogenates were clarified by centrifugation at $14000 \times g$ for $15 \mathrm{~min}$ at $4{ }^{\circ} \mathrm{C}$. The sample lysates $(80 \mu \mathrm{g}$ of protein per well) were prepared, and the proteins were separated on a $10 \%$ polyacrylamide gel. The proteins were transferred onto nitrocellulose (NC) membranes, and the blots were incubated with a blocking solution (5\% skim milk in a Tris-buffered saline and Tween-20 containing buffer) for $2 \mathrm{~h}$ at room temperature, followed by overnight incubation with primary antibodies $(1: 1000)$ at $4^{\circ} \mathrm{C}$. Immunoreactive bands were visualized by peroxidase-conjugated secondary antibodies (Jackson ImmunoResearch) and subsequent ECL-development (Pierce Biotechnology, Rockford, IL, USA). The signals were detected using a chemiluminescence detection system (BioRad, USA).

\section{Quantitative real-time RT-PCR}

Total RNA was isolated from each group of OCI-AML3 cells treated with oridonin for $24 \mathrm{~h}$ using Trizol Reagent (Invitrogen), according to the manufacturer's instructions. Two micrograms of total RNA was reverse transcribed into cDNA using the TOYOBO kits according to the manufacturer's instructions. Analysis was carried out using the SYBR Green PCR master mix (TOYOBO, Japan), and real-time RTPCR amplification was performed using an ABI Prism 7900 Sequence Detection System (Applied Biosystems, Foster City, CA, USA). All samples were performed in triplicate in three independent experiments. All threshold cycle $(\mathrm{Ct})$ values were normalized with $\beta$-actin in the same master reaction. The $2^{-\Delta \Delta \text { t }}$ (delta delta threshold cycle) analysis method was used to quantify the relative transcriptional levels of the studied genes. The specific primers used for human Nup98 were: forward, 5'-CCATTCACACCACACAGAGG-3'; reverse, 5' TGACAGGCATTCACTTCTGC-3'.

\section{Immunofluorescent staining}

The cells were exposed to various concentrations of oridonin. After $24 \mathrm{~h}$, the cells were fixed with $4 \%$ paraformaldehyde for $10 \mathrm{~min}$ and permeabilized with $0.25 \%$ Triton $\mathrm{X}-100$ for $5 \mathrm{~min}$. The cells were washed twice with PBS, blocked in 3\% bovine serum albumin and deposited onto polylysine coated coverslips. The antibodies (NPM-wt, NPM-mut and Nup98) (1:100) were incubated with non-immunoreactive IgG as a negative control. After being washed, the cells were incubated with a Cy3-labeled goat anti-rabbit secondary antibody (Pierce, Rockford, USA) diluted in PBS for $2 \mathrm{~h}$ and stained with Hoechst 33258 dye $(10 \mu \mathrm{g} / \mathrm{mL})$ for $10 \mathrm{~min}$. The images were captured and visualized using a FV500 confocal microscope (Olympus, Tokyo, Japan).

\section{Statistical analysis}

Data are presented as the mean \pm SD of at least three independent experiments. The $t$-test was used to determine the differences between treated samples and controls. A $P$ value $<0.05$ was considered significant.

\section{Results}

\section{Oridonin induces growth inhibition in OCl-AML3 cells}

We first examined the effects of oridonin on the cell viability using the MTT assay in OCI-AML3 cells. As indicated in Figure 1, oridonin exhibited a dose- and time-dependent anti-proliferative effect on OCI-AML3 cells when exposed for 24,48 , and $72 \mathrm{~h}$. The number of viable cells decreased with increasing concentrations of oridonin during the exposure time. The $\mathrm{IC}_{50}$ of oridonin for OCI-AML3 cells was 3.27 \pm 0.23 $\mu \mathrm{mol} / \mathrm{L}$ at $24 \mathrm{~h}$. As the exposure time increased, the $\mathrm{IC}_{50}$ values slightly changed. The $\mathrm{IC}_{50}$ values after 48 and $72 \mathrm{~h}$ were $3.18 \pm 0.42 \mu \mathrm{mol} / \mathrm{L}$ and $3.13 \pm 0.23 \mu \mathrm{mol} / \mathrm{L}$, respectively.

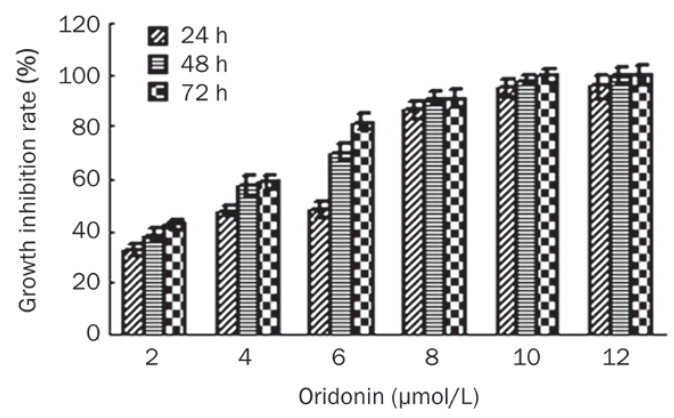

Figure 1. Oridonin induced growth inhibition in OCI-AML3 cells. OCI-AML3 cells were treated with different concentrations of oridonin as indicated for 24,48 , and $72 \mathrm{~h}$, and growth inhibition was evaluated by MTT assay. The values represent the mean \pm SD. All experiments were repeated three times with similar results.

\section{Oridonin induces apoptotic cell death in OCI-AML3 cells}

To clarify whether the observed cell death induced by oridonin was associated with the induction of apoptosis, OCIAML3 cells were incubated with various concentrations of oridonin for $24 \mathrm{~h}$, and the percentage of apoptotic and viable cells was evaluated by Annexin V FITC/PI double staining. The results in Figure 2A showed a dose-dependent increase in cells positive for Annexin V and negative for PI, indicating the onset of apoptosis in oridonin-treated cells. The apoptosis rate increased from $(6.9 \% \pm 1.2 \%)$ to $(22.5 \% \pm 3.5 \%)$ after oridonin treatment. We can conclude that the effects of oridonin on the viability of OCI-AML3 cells are mainly due to increased apoptosis and not to necrosis, as evidenced by the increased population of early-apoptotic cells. Additional evidence for increased apoptosis was the increase in the total percentage of Annexin V-positive cells, a measurement that includes secondary apoptotic cells (Figure 2B).

In addition, we observed the effects of oridonin on apoptosis in OCI-AML3 cells by Hoechst 33258 staining using laser scanning confocal microscopy. Cell nuclei in the control condition were regular in shape, but the cells were shriveled and dark-colored after oridonin treatment (Figure 2C). To determine if the pro-apoptotic effects of oridonin were accompanied by caspase activation, we examined the processing of 

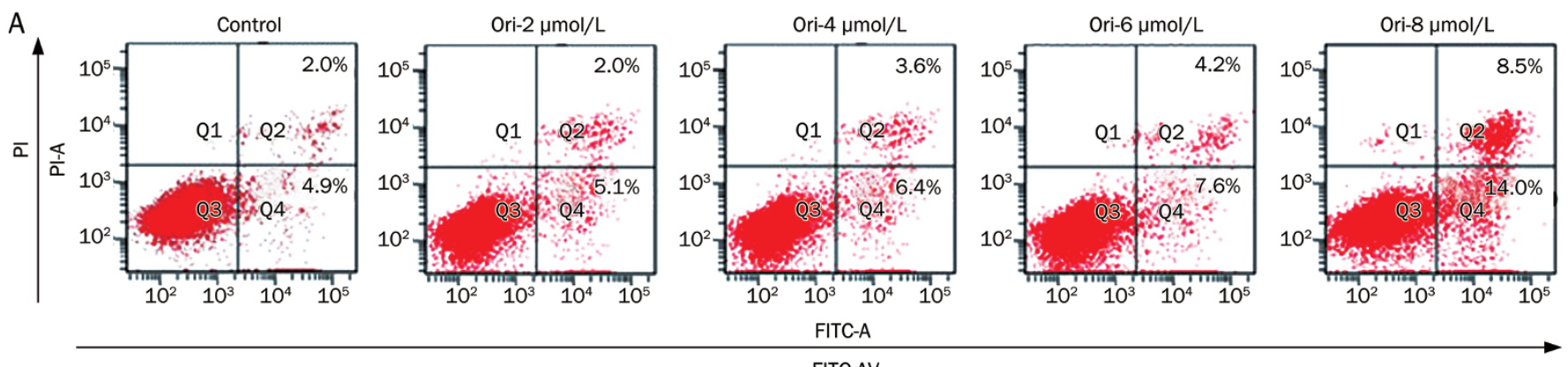

B
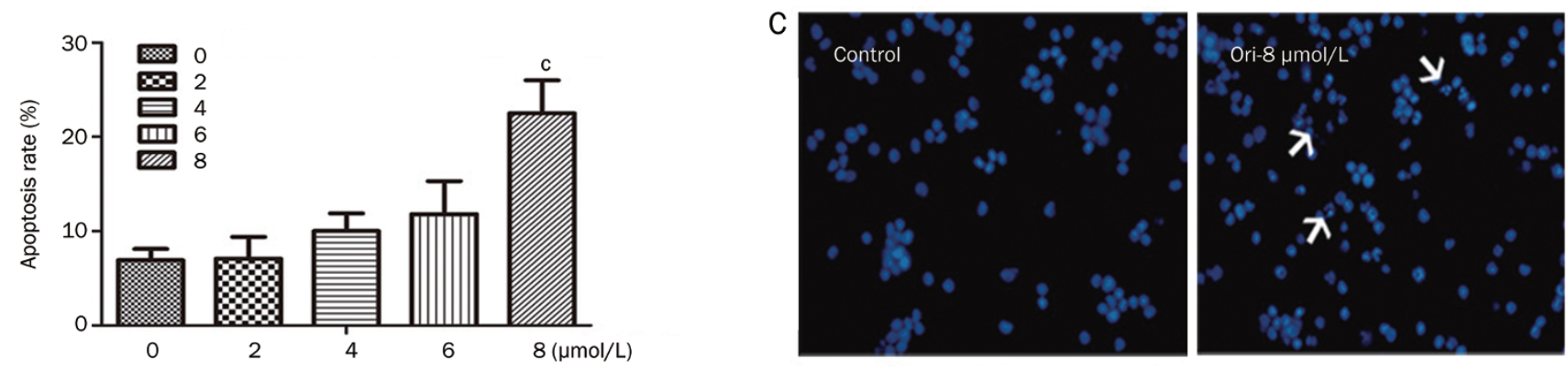

D

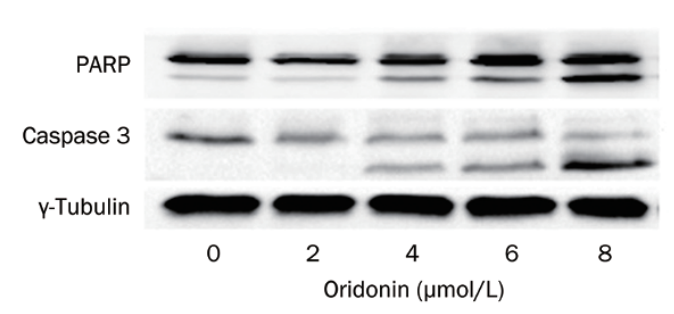

$\mathrm{E}$

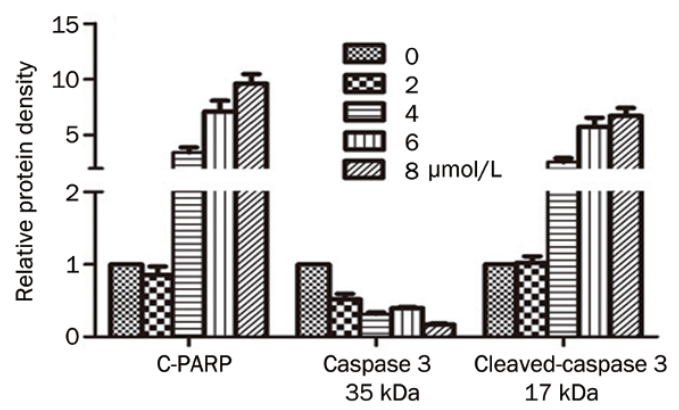

Figure 2. Oridonin induced apoptosis in OCI-AML3 cells. (A) OCI-AML3 cells were treated with different concentrations of oridonin as indicated for $24 \mathrm{~h}$, and apoptotic cells were detected by AV-PI staining using flow cytometry. The histogram shows the apoptotic rate (B). (C) OCl-AML3 cells were treated with different concentrations of oridonin as indicated for $24 \mathrm{~h}$, and apoptotic morphology was detected with a Hoechst 33258 assay. (D) After treatment with the indicated oridonin concentrations, OCI-AML3 cells lysates were blotted with PARP and caspase-3 antibodies, and $\mathrm{y}$-tubulin was used as an internal control. (E) The histogram shows the relative protein density of these proteins. The values represent the mean \pm SD. All experiments were repeated three times with similar results. ${ }^{b} P<0.05,{ }^{c} P<0.01$ indicate statistical significance compared with control cells.

caspase-3 by Western blot analysis using specific antibodies recognizing the pro-caspase and active cleavage fragments of caspase-3. The expression levels of pro-caspase-3 decreased, and the levels of cleaved caspase- 3 increased markedly in OCI-AML3 cells treated with oridonin. To further confirm the activation of caspase-3, we evaluated the cleavage of the DNA repair enzyme PARP, one of the major substrates of activated caspase-3. PARP ( $116 \mathrm{kDa})$ is cleaved into two fragments, an $\mathrm{N}$-terminal DNA-binding domain and a C-terminal catalytic domain $(85 \mathrm{kDa})$. As indicated in Figure 2D and 2E, the levels of cleaved PARP ( $85 \mathrm{kDa}$ ) increased during oridonin treatment.

Oridonin translocates NPM-mut and increases p53 protein expression

The expression of NPM-mut and NPM-wt was evaluated by
Western blot analysis using protein extracts of OCI-AML3 cells treated with various concentrations of oridonin. As indicated in Figure 3A and 3B, both the NPM-mut and NPMwt protein levels in OCI-AML3 cells were not altered after $24 \mathrm{~h}$ of treatment. Interestingly, immunofluorescent staining (Figure 3C) revealed that the NPM-mut protein translocated from cytoplasm to nucleus after incubating with oridonin for $24 \mathrm{~h}$, which was further confirmed by cytosolic/nuclear protein blotting analysis. As shown in Figure 3D, the cytoplasmic protein levels of NPM-mut decreased, but the nuclear protein levels increased after oridonin treatment. In addition, we examined the subcellular distribution of NPM-wt, as shown in Figure 3E, and NPM-wt remained in the nucleus. The recruitment of the p14arf tumor suppressor by the NPM1 mutant from the nucleolus to the nucleoplasm and cytoplasm could play a role in leukemogenesis, and furthermore, previ- 
A

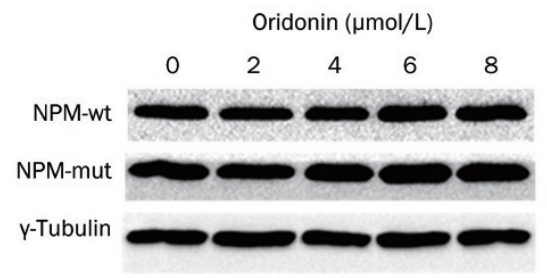

C
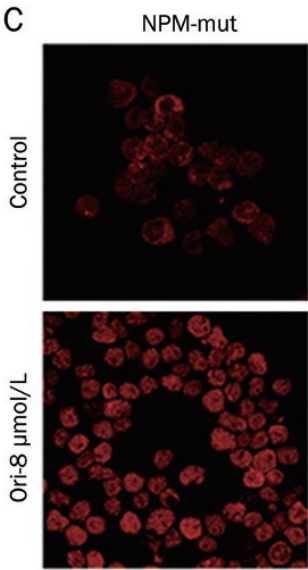

E
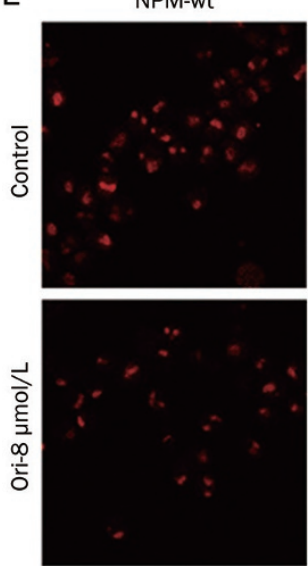

Hoechst
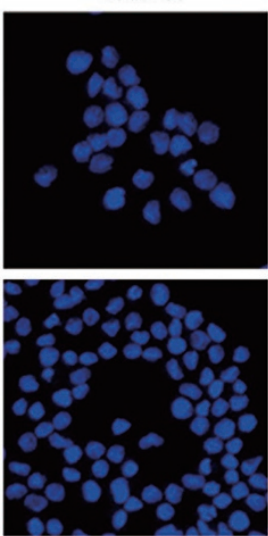

Hoechst
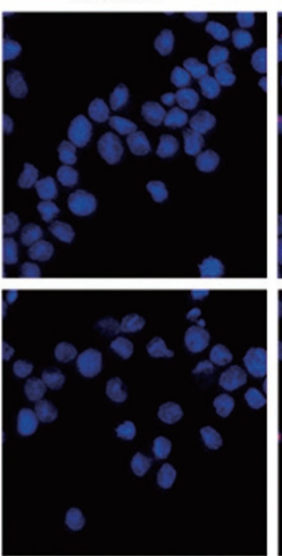
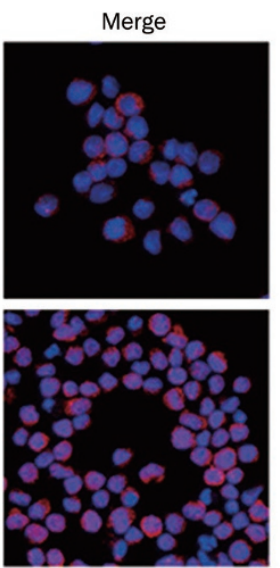

Merge
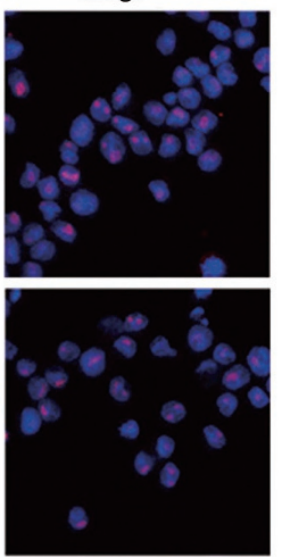

B

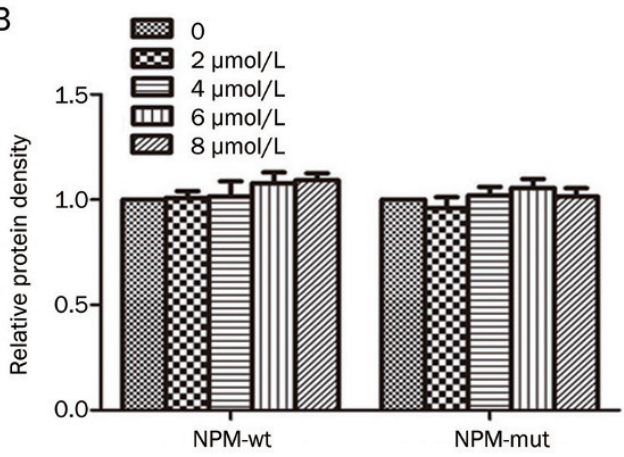

D
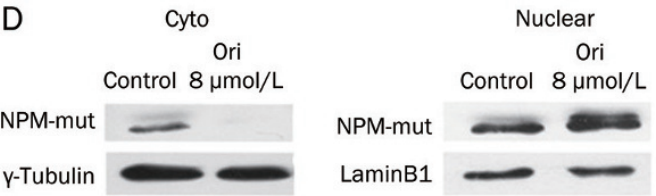

F

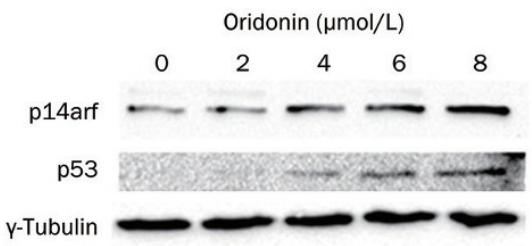

G

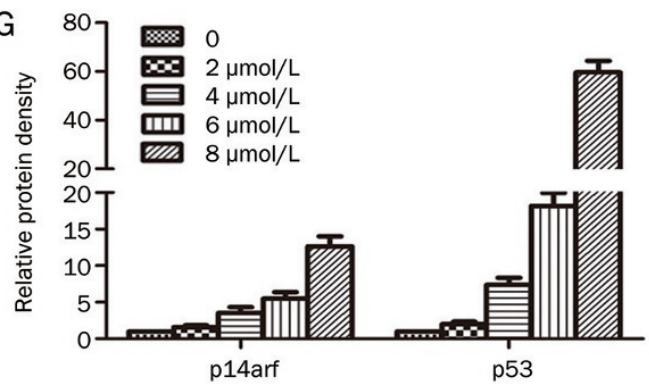

Figure 3. Effects of oridonin on the expression and localization of NPM in OCI-AML3 cells. OCI-AML3 cells were treated with increasing oridonin concentrations for $24 \mathrm{~h}$, and the relative intensities of total NPM-mut and NPM-wt protein expression were detected by Western blot (A). $\mathrm{Y}$-Tubulin was used as an internal control. (B) The histogram shows the relative protein densities of these proteins. After treatment with $8 \mu \mathrm{mol} / \mathrm{L}$ oridonin for $24 \mathrm{~h}$, OCl-AML3 cells were fixed and incubated with specific antibodies raised against NPM-mut (C) and NPM-wt (E) and followed by incubations with a Cy3labeled second antibody and Hoechst 33258. (D) Representative Western blot images of cytoplasm/nuclear NPM-mut protein, LaminB1 and $\beta$-actin, which was used as a control. (F) After treatment with different concentrations of oridonin for $24 \mathrm{~h}, \mathrm{OCl}-\mathrm{AML} 3$ cell lysates were blotted with the indicated antibodies, and $y$-tubulin was used as an internal control. (G) The histogram shows the relative protein densities of these proteins. All experiments were repeated three times with similar results.

ous research has indicated that NPM1 also interacts with p53 and influences the cellular apoptotic response ${ }^{[7]}$. Therefore, we further investigated the expression of p14arf and p53. The protein levels of p53 and p14arf increased after incubation with oridonin for $24 \mathrm{~h}$, suggesting that NPM-mut translocation might stabilize p14arf and p53 and contribute to apoptosis in OCI-AML3 cells.
Crm1 accumulates in the nucleus and Nup98 mRNA and protein levels are upregulated after oridonin treatment

The exportin Crm1 recognizes and transports proteins bearing a leucine-rich nuclear export signaling sequence. Several lines of evidence have pointed to altered Crm1-dependent nucleophosmin trafficking as the critical event in leukemogenesis in NPMc+ leukemia. To elucidate the mechanism 
A

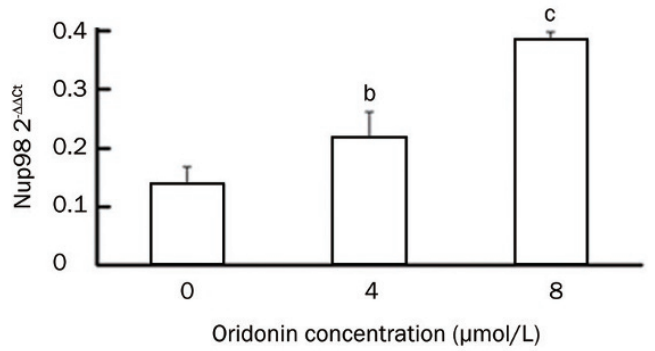

C

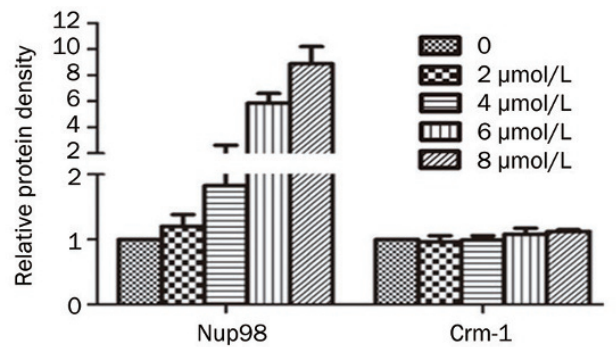

E

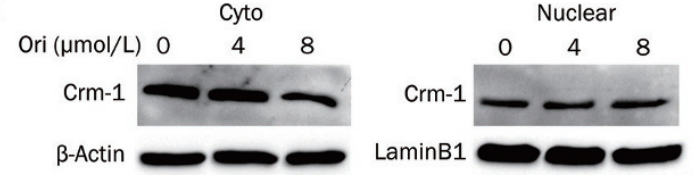

B
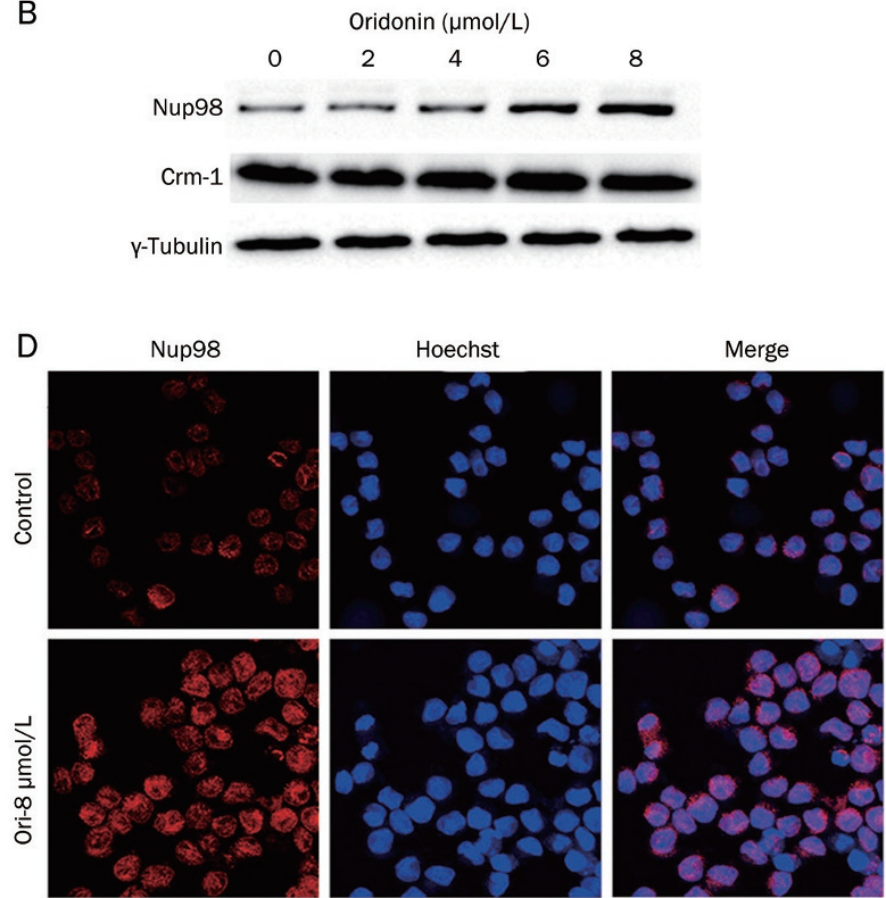

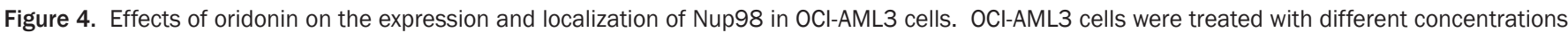

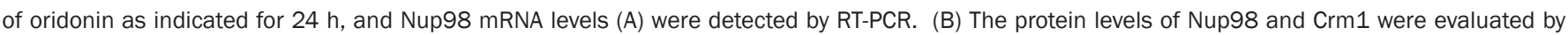

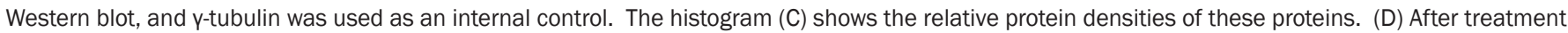

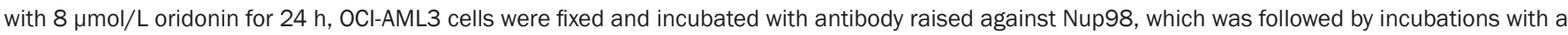

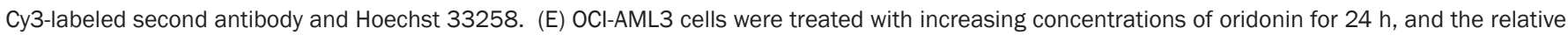

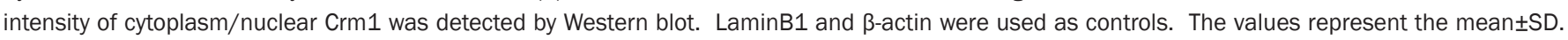
${ }^{\mathrm{b}} \mathrm{P}<0.05,{ }^{\mathrm{c}} \mathrm{P}<0.01$ compared with control cells. All experiments were repeated three times with similar results.

behind the NPM-mut redistribution induced by oridonin, we examined the expression and subcellular localization of Crm1 by Western blot. As Figure 4B indicates, the protein levels of Crm1 remained unchanged after oridonin treatment for $24 \mathrm{~h}$; however, oridonin could direct Crm1 into the nucleus (Figure $4 \mathrm{E})$. However, because Oka et al showed that Nup98 plays an important role in Crm1-mediated nuclear protein export, we then asked whether oridonin exerted its effects on OCIAML3 cells through the modulation of Nup98 expression. OCI-AML3 cells were treated with different concentrations of oridonin, and their total mRNA and protein extracts were evaluated for Nup98 expression by RT-PCR and immunoblotting, respectively. As shown in Figure 4A and consistent with the immunoblotting results (Figure $4 \mathrm{~B}$ ), the $2^{-\Delta \Delta \mathrm{Ct}}$ values of Nup98 in oridonin-treated cells were significantly increased compared with the values in untreated cells $(P<0.05, P<0.01)$. In addition, immunofluorescent staining (Figure 4D) revealed that nuclear expression of Nup98 was increased significantly after incubating with oridonin for $24 \mathrm{~h}$. The location of Crm1 from the cytoplasm to the nucleus might be associated with the upregulation of Nup98 expression.

\section{Discussion}

Mutations involving the NPM1 gene are the most frequent molecular alteration in normal karyotyped AML, accounting for $60 \%$ of cases ${ }^{[6]}$. As a consequence of these mutations, NPM1 mutant protein intracellular trafficking is altered, leading to its aberrant cytoplasmic accumulation in leukemia cells, which is considered to be the critical event in leukemogenesis $^{[2]}$. We investigated whether oridonin, a natural compound recently described to have potential as a cancer chemopreventive, was able to induce apoptosis in NPM1c+ cells and its ability to interfere with NPM mutant protein trafficking in vitro using the OCI-AML3 cell line, which can be used as a preclinical model for screening drugs and other products. Oridonin induced apoptosis in OCI-AML3 cells that was characterized by activation of caspase-3, cleavage of PARP and translocation of NPM-mut. Exposure of OCI-AML3 cells to oridonin resulted in the upregulation of p14arf and p53. We also found that the amount of Nup98 mRNA and protein was increased, which was accompanied by nuclear accumulation after treatment with oridonin. In addition, without changing the expression of Crm1, oridonin translocated Crm1 from the cytoplasm to the nucleus, suggesting that translocation of NPM-mut might be caused by the accumulation of $\mathrm{Crm} 1$ in the nucleus.

Oridonin, an ent-kaurane diterpenoid, is an important traditional Chinese herbal remedy. In this study, we evaluated if oridonin may be an effective therapeutic agent for NPMc+ 
cells. We focused on the expression of NPM-mut and NPMwt to determinate whether oridonin could modulate proteins important in promoting the leukemogenesis of NPM-mut cells. The decision to use NPM-mut and NPM-wt was based on the following observations: 1) AML with a NPM mutation has been newly identified and exhibits distinctive biological and clinical characteristics ${ }^{[6]}$; 2) In vivo and in vitro studies suggested that NPM-mut and NPM-wt play a critical role in leukemogenisis ${ }^{[2]}$. Treatment of OCI-AML3 cells with oridonin could redirect NPM-mut into the nucleus without altering the protein levels of NPM-mut and NPM-wt. Translocation of NPM-mut presumably stabilized the activity of p14arf and p53 and could facilitate apoptosis. However, the effects of oridonin on p53 might be directly influenced by itself, and further experiments are necessary to solve this problem.

Crm1, the export receptor for NES-containing proteins ${ }^{[23]}$, was found to mediate NPM-mut protein export by interacting with the new NES motif generated by the NPM mutation. Many lines of evidence point to altered Crm1-dependent nucleophosmin trafficking as the critical event in leukemogenesis $^{[24]}$. Therefore, Crm1 in AML with a NPM mutation is an attractive therapeutic target. Additionally, recent work found that Nup98 interacts with Crm1 through its N-terminal FG-repeat domain in a RanGTP-dependent manner and that Nup98 plays an important role in Crm1-mediated nuclear export $^{[25]}$, suggesting that the subcellular localization of NPMmut was co-effected by Nup98 and Crm1. Indeed, our findings suggested that the treatment of OCI-AML3 cells with oridonin upregulated the mRNA and protein levels of Nup98, which was accompanied by nuclear accumulation, and subsequently, the subcellular localization of Crm1 was altered, indicating that oridonin might influence altered nucleophosmin trafficking by retaining Crm1 in nucleus through increased Nup98 expression.

However, when NPM1 mutant cells were tested in vitro with leptomycin B, a natural product that binds irreversibly to Crm1, even the mutant protein translocated to the nucleus, and in vivo use of Crm1 inhibitors in NPM1-mutated AML does not appear to be problem free ${ }^{[24]}$. Similarly, because oridonin could re-distribute mutant NPM to the nucleoplasm, it could not direct NPM to the nucleolus (the physiological site of NPM1). Therefore, we speculate that apart from an effect on mutant NPM translocation back into the nucleus, oridonin significantly increases the expression of Nup98, p53, and p14arf, which might also contribute to apoptosis in leukemia cells.

In conclusion, oridonin may be an effective therapeutic agent in the treatment of NPMc+ leukemia cells, and thus, its study warrants further investigation.

\section{Acknowledgements}

The authors would like to thank Prof Brunangelo FALINI at the University of Perugia for kindly providing the NPMmutant specific antibody. The authors would also like to thank MD MINDEN (Ontario Cancer Institute, Toronto, ON, Canada) for providing the OCI-AML3 cells. This work was supported by grants from the National Natural Science Foundation of China (№ 81070429, 81372541, and 81060048).

\section{Author contribution}

This work was carried out in collaboration among all authors. Yan CHEN, Guo-hui CUI, Fei-fei LI, and Sha YI defined the research theme. Fei-fei LI, Sha YI, Guo-hui CUI, and Jing HE designed the methods and experiments, carried out the laboratory experiments, analyzed the data, interpreted the results and wrote the paper; Lu WEN and Li-jing YANG co-designed and co-analyzed the associated data collection and interpretation. Jing HE, Ben-ping ZHANG, and Jie ZHAO co-designed experiments, discussed analyses, interpretation, and presentation.

\section{References}

1 Falini B, Mecucci C, Tiacci E, Alcalay M, Rosati R, Pasqualucci L, et al. Cytoplasmic nucleophosmin in acute myelogenous leukemia with a normal karyotype. N Engl J Med 2005; 352: 254-66.

2 Liso A, Bogliolo A, Freschi V, Martelli MP, Pileri SA, Santodirocco M, et al. In human genome, generation of a nuclear export signal through duplication appears unique to nucleophosmin (NPM1) mutations and is restricted to AML. Leukemia 2008; 22: 1285-9.

3 Falini B, Bolli N, Liso A, Martelli MP, Mannucci R, Pileri S, et al. Altered nucleophosmin transport in acute myeloid leukaemia with mutated NPM1: molecular basis and clinical implications. Leukemia 2009; 23: 1731-43.

4 Cheng K, Sportoletti P, Ito K, Clohessy JG, Teruya-Feldstein J, Kutok JL, et al. The cytoplasmic NPM mutant induces myeloproliferation in a transgenic mouse model. Blood 2010; 115: 3341-5.

5 Bolli N, Payne EM, Grabher C, Lee JS, Johnston AB, Falini B, et al. Expression of the cytoplasmic NPM1 mutant (NPMc+) causes the expansion of hematopoietic cells in zebrafish. Blood 2010; 115: 3329-40.

6 Falini B, Nicoletti I, Martelli MF, Mecucci C. Acute myeloid leukemia carrying cytoplasmic/mutated nucleophosmin (NPMc+AML): biologic and clinical features. Blood 2007; 109: 874-85.

7 Nakagawa M, Kameoka Y, Suzuki R. Nucleophosmin in acute myelogenous leukemia. N Engl J Med 2005; 352: 1819-20; author reply 19-20.

8 Guilford JM, Pezzuto JM. Natural products as inhibitors of carcinogenesis. Expert Opin Investig Drugs 2008; 17: 1341-52.

9 Liu CX, Yin QQ, Zhou HC, Wu YL, Pu JX, Xia L, et al. Adenanthin targets peroxiredoxin I and II to induce differentiation of leukemic cells. Nat Chem Biol 2012; 8: 486-93.

10 Zhang X, Chen LX, Ouyang L, Cheng Y, Liu B. Plant natural compounds: targeting pathways of autophagy as anti-cancer therapeutic agents. Cell Prolif 2012; 45: 466-76.

11 Gao FH, Liu F, Wei W, Liu LB, Xu MH, Guo ZY, et al. Oridonin induces apoptosis and senescence by increasing hydrogen peroxide and glutathione depletion in colorectal cancer cells. Int J Mol Med 2012; 29: 649-55.

12 Ji Z, Tang Q, Zhang J, Yang Y, Liu Y, Pan Y. Oridonin-induced apoptosis in SW620 human colorectal adenocarcinoma cells. Oncol Lett 2011; 2: $1303-7$.

$13 \mathrm{Bu} \mathrm{HQ}$, Luo J, Chen H, Zhang JH, Li HH, Guo HC, et al. Oridonin enhances antitumor activity of gemcitabine in pancreatic cancer through MAPK-p38 signaling pathway. Int J Oncol 2012; 41: 949-58.

14 Cheng Y, Qiu F, Ikejima T. Molecular mechanisms of oridonin- 
induced apoptosis and autophagy in murine fibrosarcoma L929 cells. Autophagy 2009; 5: 430-1.

15 Zhang JF, Liu JJ, Liu PQ, Lin DJ, Li XD, Chen GH. Oridonin inhibits cell growth by induction of apoptosis on human hepatocelluar carcinoma BEL-7402 cells. Hepatol Res 2006; 35: 104-10.

16 Liu J, Huang R, Lin D, Wu X, Peng J, Lin Q, et al. Apoptotic effect of oridonin on NB4 cells and its mechanism. Leuk Lymphoma 2005; 46: 593-7.

17 Liu JJ, Wu XY, Lul HL, Pan XL, Peng J, Huang RW. Anti-proliferation effect of oridonin on $\mathrm{HL}-60$ cells and its mechanism. Chin Med Sci J 2004; 19: 134-7.

18 Yi S, Chen Y, Wen L, Yang L, Cui G. Downregulation of nucleoporin 88 and 214 induced by oridonin may protect OCIM2 acute erythroleukemia cells from apoptosis through regulation of nucleocytoplasmic transport of NF-kappaB. Int J Mol Med 2012; 30: 877-83.

19 Zhou GB, Kang H, Wang L, Gao L, Liu P, Xie J, et al. Oridonin, a diterpenoid extracted from medicinal herbs, targets AML1-ETO fusion protein and shows potent antitumor activity with low adverse effects on $\mathrm{t}(8 ; 21)$ leukemia in vitro and in vivo. Blood 2007; 109: 3441-50.
20 Zeng R, Chen Y, Zhao S, Cui GH. Autophagy counteracts apoptosis in human multiple myeloma cells exposed to oridonin in vitro via regulating intracellular ROS and SIRT1. Acta Pharmacol Sin 2012; 33: 91-100.

21 Zhen T, Wu CF, Liu P, Wu HY, Zhou GB, Lu Y, et al. Targeting of AML1ETO in $t(8 ; 21)$ leukemia by oridonin generates a tumor suppressor-like protein. Sci Transl Med 2012; 4: 127 ra38.

22 Quentmeier H, Martelli MP, Dirks WG, Bolli N, Liso A, Macleod RA, et al. Cell line OCl/AML3 bears exon-12 NPM gene mutation-A and cytoplasmic expression of nucleophosmin. Leukemia 2005; 19: 1760-7.

23 Kau TR, Way JC, Silver PA. Nuclear transport and cancer: from mechanism to intervention. Nat Rev Cancer 2004; 4: 106-17.

24 Falini B, Gionfriddo I, Cecchetti F, Ballanti S, Pettirossi V, Martelli MP. Acute myeloid leukemia with mutated nucleophosmin (NPM1): any hope for a targeted therapy? Blood Rev 2011; 25: 247-54.

25 Oka M, Asally M, Yasuda Y, Ogawa Y, Tachibana T, Yoneda Y. The mobile FG nucleoporin Nup98 is a cofactor for Crm1-dependent protein export. Mol Biol Cell 2010; 21: 1885-96. 